\title{
Clinical value of NT-proBNP measurements in assessing patients in the pediatric intensive care unit
}

\author{
Fang Liu ${ }^{1 \#}$, Xiaoli Luo ${ }^{2 \#} \wedge$, Xiuqi Chen ${ }^{3}$, Zhenhao $\mathrm{Lu}^{4}$, Dan Wei ${ }^{3}$, Zhiyong Yang ${ }^{3}$ \\ ${ }^{1}$ Department of Pediatrics, Maternal and Child Health Hospital of Guangxi Zhuang Autonomous Region, Nanning, China; ${ }^{2}$ Department of Pediatric \\ Critical Medicine, Chengdu Women's and Children's Central Hospital, School of Medicine, University of Electronic Science and Technology of \\ China, Chengdu, China; ${ }^{3}$ Department of Pediatrics, The First Affiliated Hospital of Guangxi Medical University, Nanning, China; ${ }^{4}$ Department of \\ Pediatrics, Nanning First People's Hospital, Nanning, China \\ Contributions: (I) Conception and design: F Liu, Z Yang, D Wei; (II) Administrative support: None; (III) Provision of study materials or patients: \\ None; (IV) Collection and assembly of data: X Chen, Z Lu; (V) Data analysis and interpretation: F Liu, Z Yang, D Wei; (VI) Manuscript writing: All \\ authors; (VII) Final approval of manuscript: All authors. \\ "These authors contributed equally to this work. \\ Correspondence to: Dan Wei. Department of Pediatrics, The First Affiliated Hospital of Guangxi Medical University, 6 Shuangyong Road, Qingxiu \\ District, Nanning, China. Email: weidanpicu@163.com; Zhiyong Yang. Department of Pediatrics, The First Affiliated Hospital of Guangxi Medical \\ University, 6 Shuangyong Road, Qingxiu District, Nanning, China. Email: 13407742223@163.com.
}

Background: N-terminal brain natriuretic peptide precursor (NT-proBNP) and brain natriuretic peptide (BNP) are mainly produced and secreted in the heart. In 2008, the European Heart Association recommended that serum BNP/NT-proBNP levels should be included in one of the diagnostic criteria of heart failure. Serum NT-proBNP is more stable than BNP, and the detection results are less affected by objective factors, so it is widely used. At present, NT-proBNP has long been beyond the scope of heart failure markers, and has a wide range of clinical value in the evaluation and prediction of some serious diseases. This study prospectively studied the predictive value of serum NT-proBNP in pediatric intensive care unit (PICU).

Methods: This was a prospective study involving 375 children in the PICU. The patients were divided into three groups: non-risk, low-risk, and high-risk groups. Serum NT-proBNP levels and the 28-day mortality rate were analyzed.

Results: The serum NT-proBNP levels and the mortality of the high-risk group was significantly higher than those of the low- and non-risk groups $(\mathrm{P}<0.01$ in both cases). Receiver operating characteristic curve (ROC curve) analysis showed that the area under the curve was $0.705(\mathrm{P}<0.001$, sensitivity $=0.643$, specificity $=0.692$ ). Death multivariate binary logistic regression analysis indicated that NT-proBNP was not an independent factor for 28-day mortality.

Conclusions: Serum NT-proBNP was significantly correlated with the severity of illness for critically ill patients in PICU. Although high levels of NT-proBNP indicated greater severity, this was not an independent risk factor affecting the prognosis of patients.

Keywords: Pediatric intensive care unit (PICU); children; N-terminal brain natriuretic peptide precursor (NT-proBNP); prediction

Submitted Feb 26, 2021. Accepted for publication May 18, 2021.

doi: $10.21037 / \mathrm{tp}-21-123$

View this article at: http://dx.doi.org/10.21037/tp-21-123

$\wedge$ ORCID: 0000-0002-0002-944X. 


\section{Introduction}

$\mathrm{N}$-terminal brain natriuretic peptide precursor (NTproBNP) is an inactive fragment of the neurohormone brain natriuretic peptide (BNP). BNP and NT-proBNP are released into the peripheral serum by cardiomyocytes in response to stress and pressure posed on the atria and ventricles. NT-proBNP is a cardiac overload biomarker associated with the severity of cardiovascular disease (1) and sepsis (2), as well as long-term physical function decline after cardiothoracic surgery (3). With the deepening of research, NT-proBNP has already gone beyond the category of heart failure diagnostic markers, and has a wide range of clinical application value in other aspects. Critical children in pediatric intensive care unit (PICU) with higher levels of NT-proBNP are common. A previous study in adult patients showed that NT-proBNP level during the acute phase of sepsis was a useful indicator of the risk of long-term impairments in physical function and muscle strength in sepsis survivors (4). Most of PICU children have infection and cardiac insufficiency. Usually, critically ill children in PICU show symptoms of cardiac insufficiency. However, its prognostic value in PICU remains unclear. The purpose of this study was to investigate the predictive value of serum NT-proBNP level on day 1 for the severity and prognosis of PICU. We present the following article in accordance with the STARD reporting checklist (available at http://dx.doi.org/10.21037/tp-21-123).

\section{Methods}

\section{Patients}

Children who were hospitalized for 24 hours or more in the First Affiliated Hospital of Guangxi Medical University during the period from July 2017 to June 2018 were selected as research subjects. The exclusion criteria were as follows: (I) those who were hospitalized during the neonatal period and transferred to the PICU due to various factors; and (II) those who were discharged and re-admitted to the PICU for various reasons within 24 hours. According to the pediatric critical illness score (PCIS), the patients were divided into a non-risk group (score of $>90$ ), a low risk group (score of 70-90), and a high-risk group (score of $<70$ ).

\section{Research methods}

The age, sex, weight, body mass index (BMI), body length, and major disease diagnosis of the patients were collected.
Blood samples were collected on the first day in the PICU. The serum NT-proBNP level $(\mathrm{pg} / \mathrm{mL})$ was detected by electrochemiluminescence in our laboratory, and the PCIS, age-adjusted Sepsis-related Organ Failure Assessment (SOFA) score, pediatric risk of mortality (PRISM) score, and the numbers of multiple organ dysfunction syndrome (MODS) were recorded on the same day. The prognosis (survival or death) of the study subjects on day 28 was followed up.

\section{Statistical analysis}

Statistical Product and Service Solutions (SPSS) 22.0 software as used for data analysis. Measurement data of normal or approximately normal distribution are expressed as mean \pm standard deviation $(\overline{\mathrm{x}} \pm \mathrm{s})$ using $t$-test or one-way analysis of variance. Non-normally distributed measurement data is represented by the median (quartile); i.e., M (P25P75), using the rank sum test. The count data is expressed as a rate (\%) using the chi-square test. The rank correlation analysis method was used for correlation analysis. Using binary logistic regression method was used to analyze the multivariate risk factors for death of children in 28 days. $\mathrm{P}<0.05$ was considered to indicate a statistically significant difference.

\section{Ethics approval and consent to participate}

All procedures performed in this study involving human participants were in accordance with the Declaration of Helsinki (as revised in 2013). The study was approved by the Ethics Committee of the First Affiliated Hospital of Guangxi Medical University and the approval number was 2017 (KY-E-101). Informed consent was obtained from the parents of the patient in this study.

\section{Results}

In total, 415 children were hospitalized for 24 hours or more in the PICU in the First Affiliated Hospital of Guangxi Medical University. 375 cases were eventually included in this study, and 40 cases were excluded.

\section{Comparison of general clinical data}

There were 127 cases in the non-risk group, including 71 males and 56 females. With a median age of 2.33 years old, a quartile age of $0.33-8.67$ years old, a median weight 
Table 1 Comparison of sex, age, weight, and BMI among the three groups

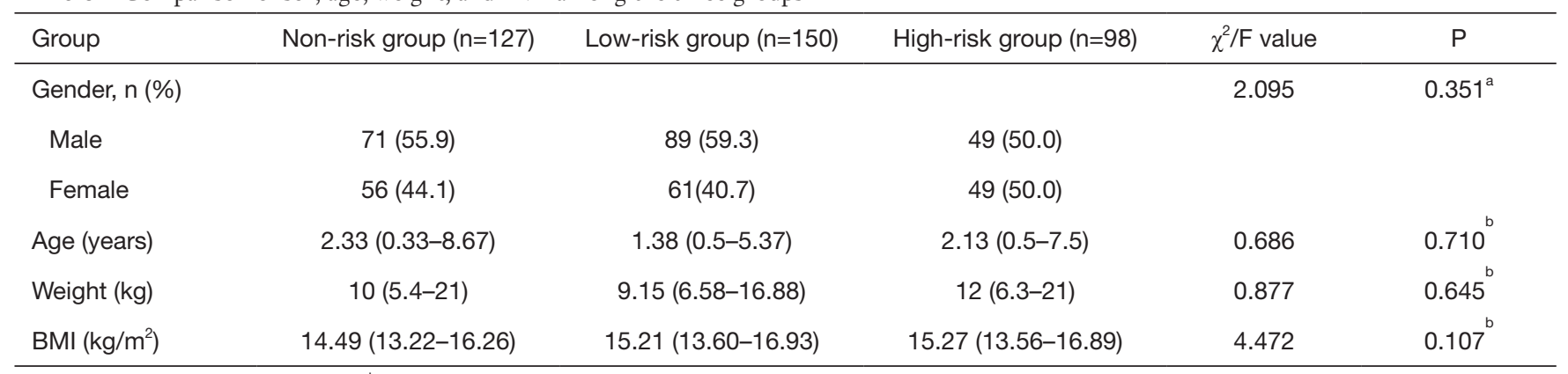

${ }^{\mathrm{a}}$, refers to the chi-square test; ${ }^{\mathrm{b}}$, refers to the rank sum test of multiple independent samples.

Table 2 Comparison of NT-proBNP levels among the three groups

\begin{tabular}{lc}
\hline Group & NT-proBNP, M (P25-P75) \\
\hline Non-risk group & $564.6(128.3-1,863)^{\star *}$ \\
Low-risk group & $722.1(250.93-2,872.5)^{\star}$ \\
High-risk group & $2,716(687.58-18,793.75)$ \\
$\chi^{2}$ & 41.765 \\
$P$ & $<0.001^{\mathrm{a}}$ \\
\hline
\end{tabular}

${ }^{a}$, the chi-square test; ${ }^{b}$, the rank sum test of two independent samples. Compared with the high-risk group, ${ }^{*} \mathrm{P}<0.001$; compared with the low-risk group, ${ }^{*} \mathrm{P}=0.013$.

Table 3 Comparison of mortality rate among three groups

\begin{tabular}{lccc}
\hline Group & $\begin{array}{c}\text { Non-survivors } \\
(\mathrm{n}=42)\end{array}$ & $\begin{array}{c}\text { Survivors } \\
(\mathrm{n}=333)\end{array}$ & $\mathrm{P}$ \\
\hline Non-risk group & $6(4.72 \%)$ & $121(95.28 \%)$ & 0.196 \\
Low-risk group & $13(8.67 \%)$ & $137(91.33 \%)$ & $<0.001$ \\
High risk group & $23(23.47 \%)$ & $75(76.53 \%)$ & $<0.001$ \\
$\chi^{2}$ & \multicolumn{2}{c}{21.156} & \\
$\mathrm{P}$ & $<0.001^{\mathrm{a}}$ & \\
\hline
\end{tabular}

of $10 \mathrm{~kg}$, a quartile weight of $5.4-21 \mathrm{~kg}$, a median BMI of 14.49 , and a quartile BMI of 13.22-16.26. There were 150 patients in the low-risk group, including 89 males and 61 females, with a median age of 1.38 years old, a quartile age of $0.5-5.37$ years old, a median weight of $9.15 \mathrm{~kg}$, a quartile weight of $6.58-16.88 \mathrm{~kg}$, a median BMI of 15.21, and a quartile BMI of 13.60-16.93. There were 98 patients in the high-risk group, including 49 males and 49 females, with a median age of 2.13 years, a quartile age of $0.5-7.5$ years, a median weight of $12 \mathrm{~kg}$, a quartile weight of $6.3-21 \mathrm{~kg}$, a median BMI of 15.27 , and a quartile BMI of 13.56-16.89. There were no significant differences in gender, age, body weight, and BMI among the three groups $(\mathrm{P}>0.05)$ (Table 1).

\section{Comparison of serum NT-proBNP levels and prognosis among groups in critical condition}

The levels of serum NT-proBNP in the three different critical condition groups were significantly different $(\mathrm{P}<0.01)$. The level of NT-proBNP in the high-risk group was significantly higher than that in the low-risk and nonrisk groups. The level of NT-proBNP in the low-risk group was significantly higher than that in the non-risk group $(\mathrm{P}<0.05)$ (Table 2).

\section{Death analysis of critically ill patients in each group within 28 days}

There were six patient deaths in the non-risk group and the mortality rate was $4.72 \%(6 / 127)$. There were three deaths in the low-risk group and the mortality rate was $8.67 \%$ (13/150). In high-risk group, there were 23 patient deaths and the mortality rate was $23.47 \%$ (23/98). The differences in the mortality rate between the three groups were statistically significant $(\mathrm{P}<0.001)$. The mortality of the high-risk group was significantly higher than that of lowrisk and non-risk groups $(\mathrm{P}<0.01)$, however, there was no notable difference in mortality between the low-risk group and non-risk group $(\mathrm{P}>0.05)$ (Table 3).

\section{ROC curve analysis of serum NT-proBNP level and severity of disease}

The area under the curve was 0.705 , and the difference 
was statistically significant $(\mathrm{P}<0.001)$. According to the curve, the critical value of serum NT-proBNP level was $1,689 \mathrm{ng} / \mathrm{mL}$, with a sensitivity of 0.643 and a specificity of 0.692 (Figure 1).

\section{Multivariate analysis of mortality within 28 days}

Multivariate binary logistic regression analysis of mortality was performed on serum NT-proBNP levels, PCT, PCIS, SOFA score, PRISM III score, mechanical ventilation time, and number of organs in MODS in the survival and death groups. The results showed that only high SOFA score was a risk factor for death of children in the PICU within 28 days $(\mathrm{P}<0.01)$, and NT-proBNP was not an independent



Figure 1 ROC curve of serum NT-proBNP levels for predicting the critical degree stage of the patient. $94 \mathrm{~mm} \times 132 \mathrm{~mm}(96 \times 96$ DPI). NT-proBNP, N-terminal brain natriuretic peptide precursor. factor for 28-day mortality (Table 4).

\section{Discussion}

BNP can dilate blood vessels, inhibit vascular smooth muscle and myocardial fibrosis, regulate blood pressure, promote natriuresis, inhibit sympathetic nervous system activity, and antagonize the renin-angiotensin-aldosterone system (5). There are numerous reports indicating that BNP/NT-proBNP play important roles in some diseases (other than cardiovascular illnesses), such as judging the severity of the disease, evaluating the therapeutic effect, predicting poor prognosis, etc. (5-7). Compared with BNP, NT-proBNP has stronger stability, longer half-life period, less individual variation, is less affected by environmental factors, and the former does not have biological activity. Thus, the serum NT-proBNP level is widely used in clinical practice to reflect BNP levels.

NT-proBNP can be used as a serological indicator to assess the severity of critically ill children, and has a significant negative correlation with the PCIS score (8). This study also found that the higher the level of serum NT-proBNP, the lower the PCIS score, and the more serious the condition is. The elevation of NT-proBNP in critically ill children is primarily associated with increased secretion and metabolic disorders. NT-proBNP is mainly synthesized and secreted in the ventricle. Increased ventricular load and tension of the ventricular wall are the main conditions that stimulate ventricular secretion of NTproBNP (9).

Related reports indicate that hypoxia-ischemia, microcirculatory disorders, oxidative stress, and stimulation of pro-inflammatory factors lead to increased cardiac secretion of NT-proBNP (10). The only mode of removal of NT-proBNP is glomerular filtration, the level of which is

Table 4 Binary logistical regression analysis of the 28-day mortality endpoint

\begin{tabular}{lcccccc}
\hline Index & B & S.E. & Wald & P & OR & 95\% confidence interval \\
\hline NT-proBNP & 0.000 & 0.000 & 0.109 & 0.741 & 1.000 & $1.000-1.000$ \\
PCT & -0.005 & 0.007 & 0.620 & 0.431 & 0.995 & $0.981-1.008$ \\
Mechanical ventilation time & 0.000 & 0.001 & 0.077 & 0.782 & 1.000 & $0.998-1.002$ \\
PCIS score & -0.013 & 0.035 & 0.138 & 0.710 & 0.987 & $0.921-1.057$ \\
PRISM III score & 0.068 & 0.036 & 3.663 & 0.056 & 1.071 & $0.998-1.148$ \\
SOFA score & 0.233 & 0.084 & 7.721 & 0.005 & 1.263 & $1.071-1.489$ \\
Number of MODS & 0.323 & 0.282 & 1.304 & 0.253 & 1.381 & $0.794-2.402$ \\
\hline
\end{tabular}


mainly affected by renal function (11), and is not affected by the natriuretic peptide receptor. Some studies also found that elevated serum NT-proBNP in hyponatremia is caused by craniocerebral diseases, such as infection and bleeding (12). The present study found that the mortality rate of the critically ill group was significantly higher than that of lowrisk and non-risk groups, suggesting that the PICS score is related to the mortality rate. Zhang et al. and other studies also found that the PICS score can better predict the mortality of critically ill children (8).

In addition, the area under the ROC curve of NTproBNP and the critically ill condition was 0.705 , suggesting that it has a good effect on the judgment of extremely critical illness. However, with a critical value of $1,689 \mathrm{ng} / \mathrm{mL}$, its specificity and sensitivity were normal, which was considered to be related to the large number of diseases mentioned in this paper.

In this paper, it was shown that serum NT-proBNP levels were significantly positively correlated with inflammatory markers [C-reactive protein (CRP), Procalcitonin (PCT)], suggesting that serum NT-proBNP levels are associated with inflammatory factors. BNP has anti-inflammatory and anti-fibrotic effects. The pro-inflammatory response in the bodies of patients with sepsis and the toxicity of the pathogen to the heart increases the secretion of BNP/NTproBNP, while the rejection of NT-proBNP is severely blocked in septic shock, and its level is often high.

This study also showed that serum NT-proBNP was significantly positively correlated with SOFA scores, and studies have found that SOFA scores have a high clinical value in predicting mortality $(13,14)$, suggesting that serum NT-proBNP may be helpful in assess prognosis. Furthermore, serum NT-proBNP levels, mechanical ventilation time, SOFA score, PRISM III score, and MODS were found to be significantly higher in the death group than in the survival group, and the PCIS score was significantly lower in the death group than the survival group. These six indicators maintain a certain degree of value to predict 28 -day mortality and the prognosis of children.

The multivariate binary logistic regression of mortality showed that only high SOFA score was a risk factor for mortality of PICU children within 28 days. However, whether NT-proBNP can be used as a predictor of 28-day prognosis requires dynamic monitoring of NT-proBNP levels and supporting evidence from larger samples. Previous studies have shown that serum NT-proBNP can assist in the diagnosis of sepsis, and can also assess the condition and prognosis (15).

However, this study failed to dynamically monitor serum NT-proBNP levels, and therefore, the prognosis prediction of children in the PICU could not be comprehensively determined. In future, thorough study of the level of serum NT-proBNP is needed in order to determine the predictive value of serum NT-proBNP more fully.

\section{Conclusions}

In conclusion, serum NT-proBNP is associated with a critical condition in patients. High levels of serum NTproBNP suggest that the child's condition is severe, which is useful in predicting the severity of PICU children, however it is not an independent risk factor for prognosis. Considering that this is a single-center study, and there is no study on the impact of serum NT-proBNP level changes on the condition and prognosis of children, and the numbers of patients included in the research was relatively small, more large-scale clinical research is required to validate the conclusions of this study.

\section{Acknowledgments}

The authors would like to thank Dr. Dev Sooranna, Imperial College London, for editing the manuscript. Funding: None.

\section{Footnote}

Reporting Checklist: The authors have completed the STARD reporting checklist. Available at http://dx.doi.org/10.21037/ tp-21-123

Data Sharing Statement: Available at http://dx.doi. org/10.21037/tp-21-123

Conflicts of Interest: All authors have completed the ICMJE uniform disclosure form (available at http://dx.doi. org/10.21037/tp-21-123). The authors have no conflicts of interest to declare.

Ethical Statement: The authors are accountable for all aspects of the work in ensuring that questions related to the accuracy or integrity of any part of the work are appropriately investigated and resolved. All procedures performed in this study involving human participants were in accordance with the Declaration of Helsinki (as revised in 
2013). The study was approved by the Ethics Committee of the First Affiliated Hospital of Guangxi Medical University and the approval number was 2017 (KY-E-101). Informed consent was obtained from the parents of the patient in this study.

Open Access Statement: This is an Open Access article distributed in accordance with the Creative Commons Attribution-NonCommercial-NoDerivs 4.0 International License (CC BY-NC-ND 4.0), which permits the noncommercial replication and distribution of the article with the strict proviso that no changes or edits are made and the original work is properly cited (including links to both the formal publication through the relevant DOI and the license). See: https://creativecommons.org/licenses/by-nc-nd/4.0/.

\section{References}

1. Chow SL, Maisel AS, Anand I, et al. Role of Biomarkers for the Prevention Assessment and Management of Heart Failure: A Scientific Statement From the American Heart Association. Circulation 2017;135:e1054-91.

2. Turner KL, Moore LJ, Todd SR, et al. Identification of cardiac dysfunction in sepsis with B-type natriuretic peptide. J Am Coll Surg 2011;213:13946; discussion 146-7.

3. Fox AA, Marcantonio ER, Collard CD, et al. Increased peak postoperative B-type natriuretic peptide predicts decreased longer-term physical function after primary coronary artery bypass graft surgery. Anesthesiology 2011;114:807-16.

4. Custodero C, Wu Q, Ghita GL, et al. Prognostic value of NT-proBNP levels in the acute phase of sepsis on lower long-term physical function and muscle strength in sepsis survivors. Crit Care 2019;23:230.

5. Roubinian NH, Looney MR, Keating $\mathrm{S}$, et al. Differentiating pulmonary transfusion reactions using recipient and transfusion factors. Transfusion 2017;57:1684-90.

Cite this article as: Liu F, Luo X, Chen X, Lu Z, Wei D, Yang Z. Clinical value of NT-proBNP measurements in assessing patients in the pediatric intensive care unit. Transl Pediatr 2021;10(5):1355-1360. doi: 10.21037/tp-21-123
6. Baek JY, Song MS. Meta-analysis of factors predicting resistance to intravenous immunoglobulin treatment in patients with Kawasaki disease. Korean J Pediatr 2016;59:80-90.

7. Deng HL, Zhang YF, Li YP, et al. N-terminal pro-brain natriuretic peptide level associated with severe hand, foot and mouth disease. BMC Infect Dis 2016;16:585.

8. Zhang L, Huang H, Cheng Y, et al. Predictive value of four pediatric scores of critical illness and mortality on evaluating mortality risk in pediatric critical patients. Zhonghua Wei Zhong Bing Ji Jiu Yi Xue 2018;30:51-6.

9. Volpe M, Battistoni A, Rubattu S. Natriuretic peptides in heart failure: Current achievements and future perspectives. Int J Cardiol 2019;281:186-9.

10. Chiba A, Watanabe-Takano H, Miyazaki T, et al. Cardiomyokines from the heart. Cell Mol Life Sci 2018;75:1349-62.

11. Sinha A, Gopinathan P, Chung YD, et al. Simultaneous detection of multiple NT-proBNP clinical samples utilizing an aptamer-based sandwich assay on an integrated microfluidic system. Lab Chip 2019;19:1676-85.

12. Misra UK, Kalita J, Kumar M, et al. A study of atrial and brain natriuretic peptides in tuberculous meningitis and acute encephalitis. Int J Tuberc Lung Dis 2018;22:452-7.

13. Wang XW, Niu XG, Li JX, et al. SOFA Score Can Effectively Predict the Incidence of Sepsis and 30-Day Mortality in Liver Transplant Patients: A Retrospective Study. Adv Ther 2019;36:645-51.

14. Schlapbach LJ, Straney L, Bellomo R, et al. Prognostic accuracy of age-adapted SOFA, SIRS, PELOD-2, and qSOFA for in-hospital mortality among children with suspected infection admitted to the intensive care unit. Intensive Care Med 2018;44:179-88.

15. Varpula M, Pulkki K, Karlsson S, et al. Predictive value of $\mathrm{N}$-terminal pro-brain natriuretic peptide in severe sepsis and septic shock. Crit Care Med 2007;35:1277-83.

(English Language Editor: A. Kassem) 